\title{
An IPA Study Exploring the Experiences of Body Image in Women, Post-Pregnancy
}

\author{
L. Newson ${ }^{1}$, C. Mission ${ }^{1}$ and J.C Abayomi ${ }^{2}$ \\ ${ }^{1}$ Faculty of Science, Liverpool John Moores University, City Campus, Byrom Street, Liverpool, L3 3AF, UK and \\ ${ }^{2}$ Faculty of Education, Community and Leisure, Liverpool John Moores University, UK
}

Previous research suggests that postpartum women have both positive and negative body image ${ }^{(1)}$. Body image experiences in postpartum women have been explored in Turkish, Taiwanese and Japanese cultures ${ }^{(2,3)}$. However, within the UK, body image has been researched using quantitative methods and predominantly adolescent samples.

This study used a qualitative approach to gain a deeper understanding of the experiences and perspectives of body image, in women during their postpartum period within the Western UK culture. Convenience sampling was used to recruit eight women (mean age 31.25) within their first year postpartum (range 3-9 months) from a children centre, North West England. Qualitative methodology was utilised to explore the experiences of body image in women during their postpartum period. Semi-structured interviews were conducted, in a quiet, private room, lasting approximately 30-45 minutes and analysed using the interpretative phenomenological approach $^{(4)}$. Four themes were identified: 1) Support, 2) Altered perspective,

"I was seeing my body in terms of what it can do, and its power... And it's beauty as a mother. So I didn't care what I looked like... I see its function, I don't just feel like I'm supposed to look this way, to be like this, I feel proud of my body and what it's done and what it's achieved"

3) Influence of society /media and 4) physical body appearance.

“... cause I'm conscious of, there's all sorts of stuff about getting your body back and do you fit into your pre-pregnancy clothes... I think there is a bit of pressure to of pressure to sort of... yeah get your body back to how it was again"

The postpartum period is a time of change and transition for women, and is influenced by individual pregnancy experiences, individual support circles, society and media. Women engage in social comparisons, either to evaluate themselves or motivate their own progression. Postpartum women may experience a loss of self, when being consumed by the new mother role they are occupying, and may feel conflicted in which social expectations they should be attaining. Therefore, postpartum women should be provided with the knowledge and support that enables them to transition positively into their new role, whilst simultaneously allowing them to improve their body image, in a healthy manner. The media portrayal of the ideal postpartum body should emphasise the need for women to appreciate their new bodies, celebrate their power and enjoy time with their babies, instead of hindering body image by emphasising celebrity post-pregnancy progress. Although some forms of media have used interviews with new mothers to allow a deeper insight into the postpartum period, this is rare. The importance of individual support circles needs to be emphasised, and partners in particular should be provided with information about how to best aid the transition from pregnancy to motherhood.

1. Silveira ML, Ertel KA, Dole N \& Chasan-Taber L (2015). Arch Womens Ment Health 18(3), 409-421.

2. Chang S, Chao YY \& Kenney NJ (2006). Birth 33(2), 147-153.

3. Fuller-Tyszkiewicz M, Skouteris H, Watson BE \& Hill B. (2012) Journal of Health Psychology, 0(0), 1-11.

4. Smith JA, Flowers P \& Larkin M (2009) Interpretative Phenomenological Analysis - Theory, Method, Research, Los Angeles; London: Sage. 